\title{
Exosomes derived from human amniotic fluid mesenchymal stem cells alleviate cardiac fibrosis via enhancing angiogenesis in vivo and in vitro
}

\author{
Jiajia Hu ${ }^{1 \#}$, Xuliang Chen ${ }^{2 \#}$, Ping $\mathrm{Li}^{3,4}$, Xiaoxu Lu ${ }^{1}$, Jianqin Yan $^{1}$, Huiling $\operatorname{Tan}^{5}$, Chengliang Zhang \\ ${ }^{1}$ Department of Anesthesiology, Xiangya Hospital, Central South University, Changsha, China; ${ }^{2}$ Department of Cardiovascular Surgery, Xiangya \\ Hospital, Central South University, Changsha, China; ${ }^{3}$ Department of Obstetrics, Xiangya Hospital, Central South University, Changsha, China; \\ ${ }^{4}$ Hunan Engineering Research Center of Early Life Development and Disease Prevention, Changsha, China; ${ }^{5}$ Department of Anesthesiology, Hunan \\ Provincial People's Hospital, The First Affiliated Hospital of Hunan Normal University, Changsha, China \\ Contributions: (I) Conception and design: C Zhang, P Li; (II) Administrative support: X Chen, J Yan; (III) Provision of study materials or patients: J \\ Hu, H Tan, X Lu; (IV) Collection and assembly of data: P Li, X Lu; (V) Data analysis and interpretation: X Chen, C Zhang; (VI) Manuscript writing: \\ All authors; (VII) Final approval of manuscript: All authors. \\ \#These authors contributed equally to this work. \\ Correspondence to: Chengliang Zhang, MD. Department of Cardiovascular Surgery, Xiangya Hospital, Central South University, 87 Xiangya Road, \\ Changsha 410008, China. Email: zhangchengliang@csu.edu.cn.
}

Background: Cardiac fibrosis is a pathological process characterized by excess extracellular matrix (ECM) deposition and plays a critical role in nearly all types of heart disease. The mechanism of cardiac fibrosis is still unclear and no effective medication treatment of cardiac fibrosis. Research showed that mesenchymal stem cell (MSC) derived exosomes may play a critical role in cardiac fibrosis. The effect of human amniotic fluid MSC (hAFMSC)-derived exosomes (hAFMSCExos) on cardiac fibrosis has remained unclear.

Methods: The hAFMSCExos were extracted using a sequential centrifugation approach. The effects of hAFMSCExos on angiogenesis were analyzed both in human umbilical vein endothelial cells (HUVECs) after oxygen and glucose deprivation (OGD) in vitro, and in isoproterenol (ISO) induced-cardiac fibrosis in vivo.

Results: The hAFMSCExos remarkably up-regulate the motility and migration of HUVECs after OGD compared with phosphate-buffered saline (PBS). Meanwhile, total tube length, total branching points and total loops were significantly raised in HUVECs after OGD treated with hAFMSCExos. The hAFMSCExos alleviated the cardiac fibrosis degree tested by hematoxylin-eosin (H\&E) and Masson staining. The protein levels of Collagen I and $\alpha$-smooth muscle actin ( $\alpha$-SMA) were lower in exosomes group rats than PBS group. Immunofluorescence suggested that hAFMSCExos can promote the expression of CD31 in the rats. Meanwhile, the number of regenerated microvessels was significantly enhanced in rats administrated with exosomes by quantitative analysis of microvessel density. Furthermore, the micro-CT scanning evidenced that hAFMSCExos promote angiogenesis after cardiac fibrosis. The levels of hypoxia-inducible factor $1 \alpha$ (HIF-1 $\alpha$ ) and vascular endothelial growth factor (VEGF) expression in the left ventricle accepted HUVECs were higher than PBS treatment at 7 days post-treatment by Western blot analysis.

Conclusions: The hAFMSCExos have proangiogenic effects on endothelial cells and enhanced angiogenesis in cardiac fibrosis. The hAFMSCExos may be a promising potential treatment strategy for cardiac fibrosis.

Keywords: Human amniotic fluid; mesenchymal stem cells (MSCs); exosome; cardiac fibrosis; angiogenesis

Submitted Sep 08, 2020. Accepted for publication Feb 26, 2021.

doi: $10.21037 / \mathrm{cdt}-20-1032$

View this article at: http://dx.doi.org/10.21037/cdt-20-1032 


\section{Introduction}

Cardiac fibrosis is a pathological process characterized as excess extracellular matrix (ECM) deposition and fibroblasts transdifferentiate into myofibroblasts (1). Excessive cardiac fibrosis plays a critical role in nearly all forms of heart disease such as myocardial infarction (MI), heart failure (HF), atrial fibrillation, hypertensive heart disease, diabetic cardiomyopathy, and idiopathic dilated cardiomyopathy (2). The cardiac fibrosis level was found to be a powerful predictor of death and adverse cardiac events in HF patients $(3,4)$.

The mechanism of cardiac fibrosis is still unclear. ECM deposition and cardiac fibroblasts $(\mathrm{CFs})$ transdifferentiation play a critical role in cardiac fibrosis $(5,6)$. The adult mammalian heart has a poor regenerative ability; thus, the damage and loss of numerous cardiomyocytes trigger the fibroblasts to activate into myofibroblasts and start the reparative program. Unfortunately, no effective medication treatment could rescue the fibrotic cardiac tissue, due to the lack of self-regeneration capability of the cardiomyocyte. It is urgent to pursue effective therapeutic strategies for cardiac fibrosis.

Fortunately, more recent evidence showed that exosomes derived from mesenchymal stem cells (MSCs) have the potential of effective therapeutic strategies, and exosomes derived from MSCs were the primarily effective factor of classical MSC-based therapy (7-10). Meanwhile, exosomes derived from MSCs showed similar therapeutic effects and functional properties as MSCs without apparent adverse effects $(11,12)$. Exosomes are highly contributed to the cardiac repair and regeneration with the biological antiapoptotic, anti-fibrotic, and pro-angiogenic characteristics (12-15). Moreover, exosomes treatment has distinctive advantages: without engraftment requirement, which reduces concerns for tumorigenesis (16), and their nanoscale level enhances the penetrability of vessels, barriers and bio-membranes (17). Collectively, exosomes recapitulate to a large extent the immensely broad therapeutic effects previously attributed to MSCs (18).

Reports confirmed that exosomes can enhance cardiac repair and attenuate the level of cardiac fibrosis $(19,20)$. Exosomes exert key functions for cell differentiation, proliferation, cell death, intercellular communication, angiogenesis, epigenetics, immunomodulation, and gene regulation (21-23). Exosomes are stable in target tissues with a long duration and can transmit extracellular signals molecules intracellularly (21). Human amniotic fluid
MSCs (hAFMSCs) are isolated from leftover samples of amniotic fluid, via amniocentesis screening (24) or cesarean section (25). The research demonstrated that the hAFMSCs had cardioprotective paracrine potential in myocardial ischemia/reperfusion injury rats (26).

We hypothesized that hAFMSC-derived exosomes (hAFMSCExos) have a therapeutic effect on cardiac fibrosis. To test this hypothesis, we examined the application of the hAFMSCExos as a regenerative medicine both in isoproterenol (ISO)-induced rat cardiac fibrosis model and in human umbilical vein endothelial cells (HUVECs), and aimed to elucidate the potential regulatory mechanism of hAFMSCExos on cardiac fibrosis by regulating angiogenesis. We present the following article in accordance with the ARRIVE reporting checklist (available at http:// dx.doi.org/10.21037/cdt-20-1032).

\section{Methods}

All the experiment processes were showed in Figure S1.

\section{Isolation, cultured and identification hAFMSC}

The human amniotic fluid was obtained from healthy mothers in the cesarean section between 32- and 36-week gestation without $\mathrm{HIV}$, hepatitis $\mathrm{B}$, and hepatitis $\mathrm{C}$, and the collection was performed in compliance with the Helsinki declaration.

HAFMSCs were isolated from amniotic fluid based on methods as previously described by Tracy et al. (16). The light microscope was used to evaluate the colonyforming capacity and the sharpness of hAFMSCs. The differentiation abilities of hAFMSCs were assessed by cultured with osteogenic, adipogenic, and chondrogenic differentiation media (hMSC Differentiation BulletKit; Lonza, USA). Alizarin Red staining, Oil Red O staining, and Alcian Blue staining were selected to identify differentiated cells after differentiation culture. The phenotype profile of hAFMSCs was identified by flow cytometry, and the cell surface molecular markers including CD29, CD34, CD44, CD45 and CD105 were tested.

\section{Isolation and identification of hAFMSCExos}

Exosomes were prepared using sequential centrifugation according to methods described previously (27). The exosomes were isolated from the supernatants of hAFMSCs using ExoQuick-TC Kit (System Biosciences, 
USA) in accordance with manufacturer's instructions. The hAFMSCs were cultured in a conditioned medium containing 10\% exosome-free fetal bovine serum (FBS) for 48 hours for the preparation of exosomes isolation, and then the supernatants of hAFMSCs were collected by initial centrifugation at $3,000 \mathrm{~g}$ for 15 minutes to pellet and remove the cell debris, then followed by sequential centrifugations at $13,000 \mathrm{~g}$ for $30 \mathrm{~min}$, followed by centrifugation at 100,000 $\mathrm{g}$ for $60 \mathrm{~min}$ (Himac CS150GXII, HITACHI, Japan). Then the exosome-enriched fraction was resuspended in phosphate-buffered saline (PBS). To identify the hAFMSCExos, exosomes resuspended in $0.1 \mathrm{~mL}$ of PBS was used for a transmission electron microscope and particle size analysis, and exosomes resuspended in $0.1 \mathrm{~mL}$ RIPA buffer for protein quantification and Western blotting. $0.5 \times 10^{9}$ exosomes were administered to cultured cells in vitro and $5 \times 10^{9} / 40 \mu \mathrm{L}$ to experiment rats in vivo to evaluate the function of hAFMSCExos. Besides, the morphologies of exosomes were observed with a transmission electron microscope (Hitachi H-7100 microscope; HITACHI, Japan).

\section{HUVEC lines culture}

HUVECs (China Cell Culture Center, Shanghai, China) were cultured in Roswell Park Memorial Institute (RPMI) 1640 medium (Gibco, USA) with 10\% FBS and $100 \mathrm{U} / \mathrm{mL}$ penicillin. All HUVECs were maintained at $37{ }^{\circ} \mathrm{C}$ with $5 \%$ $\mathrm{CO}_{2}$ in a humidified atmosphere and incubated overnight. All experiments were performed before passage 7 .

\section{Exosomes uptake assay in vitro}

Exosomes uptake assay was performed to evaluate whether HAFMSCExos can be absorbed by HUVECs in vitro and the heart of rats in vivo. The PKH26 dye (PKH26, Sigma) can label the lipid membranes of HAFMSCExos with a red fluorescent. After incubated at $37^{\circ} \mathrm{C}$ for $30 \mathrm{~min}$, the PKH26-labeled suspension was centrifuged at $300 \mathrm{~g}$ for $15 \mathrm{~min}$, and washed twice with PBS. Then seeded into culture flasks for incubation for $48 \mathrm{~h}$. Next, HUVEC lines were incubated with HAFMSCExos which have been labeled with the red fluorescent dye at $37^{\circ} \mathrm{C}$ for $3 \mathrm{~h}$. Then HUVECs were labeled with DAPI (Invitrogen, USA) and CD31 (Invitrogen, USA) after washed with PBS and fixed with $4 \%$ paraformaldehyde for $15 \mathrm{~min}$ lastly. Exosomes uptake by HUVECs were analyzed by fluorescent signal analysis with a fluorescence microscope (Leica DMI6000B,
Germany).

\section{Oxygen and glucose deprivation (OGD) model}

The HUVECs were cultured in the glucose-free DMEM (Gibco) medium with $94 \% \mathrm{~N}_{2}, 1 \% \mathrm{O}_{2}$ and $5 \% \mathrm{CO}_{2}$ for $4 \mathrm{~h}$ at $37^{\circ} \mathrm{C}$. HUVECs were washed with RPMI 1640, and recovered in normal culture medium under normoxic incubation conditions for 12-48 h. Then these HUVECs subjected to OGD were divided into two groups, including a control group and exosomes treatment group.

\section{Transwell chamber migration assay}

To detect the migration of HUVECs, the transwell migration assay was analyzed as previously described (28). In brief, HUVECs were plated into the upper chamber of the 24-well transwell migration chamber with $8 \mu \mathrm{m}$ filter inserts (BD Biosciences, USA). HUVECs with $1 \times 10^{4}$ cells per well were suspended and plated into the upper chamber containing a serum-free DMEM medium. Approximately $500 \mu \mathrm{L}$ complete medium supplemented with HAFMSCExos $(100 \mu \mathrm{g} / \mathrm{mL})$ in the exosomes group or an equal volume of PBS in the PBS group was added to the lower chamber. After $12 \mathrm{~h}$ of incubation for HUVECs, the non-migrating cells were removed thoroughly with a cotton swab, and the migrated and invasive cells on the bottom side of the filter were fixed with $100 \%$ methanol for $30 \mathrm{~min}$. Then cells were stained with $0.1 \%$ crystal violet for $20 \mathrm{~min}$. The number of the migrated HUVECs was quantified in at least three random fields under an optical microscope at a $\times 100$ magnification (Leica).

\section{Scratch wound migration assay}

Scratch wound migration assay was performed to detect the migration of HUVECs. The scratch wound migration assay was performed as previously described (28). In brief, HUVECs were cultured in a 6-well plate in normal growth media and incubated overnight.

A sterile $200-\mu \mathrm{L}$ pipette tip was selected to make scratches. Two parallel scratches were made within the cell monolayer for each well, and made the cell confluence reach approximately $90 \%$. The cells were washed with PBS to remove the floating HUVECs. The adhered HUVECs were treated with hAFMSCExos $(100 \mu \mathrm{g} / \mathrm{mL})$ or an equal volume of PBS. After the cell recovered by incubated for $12 \mathrm{~h}$, images of scratch wound migration were visualized 
with a phase-contrast microscope (CKX41; Olympus, Japan). The quantitative analysis was performed to examine the migrated area from the border of the scratch was quantified by using the Image-Pro Plus software (Media Cybernetics, USA).

\section{Tube formation assay}

The effect of hAFMSCExos on HUVECs angiogenesis in vitro was examined by tube formation assay. The Vitroangiogenesis Assay Tube Formation Kit (Cultrex ${ }^{\circledR}$, USA) was used in the tube formation assay. Ninety-six-well plates were pre-coated with $50 \mu \mathrm{L}$ Matrigel per well at $2 \times 10^{4}$ cells/well, and HUVECs were resuspended in the precoated plates, and the. Conditioned medium with exosomes $(100 \mu \mathrm{g} / \mathrm{mL})$ or PBS was also added to well for incubation at $37^{\circ} \mathrm{C}$ for $12 \mathrm{~h}$, capillary-like tube formation was detected under an inverted microscope (Leica). Quantitative analyses of the network structure including total branching points, tube length, and loops were performed using the Image-Pro Plus software (Media Cybernetics, USA).

\section{Cardiac fibrosis model establishment, and exosomes treatment}

Healthy adult SD rats (weight 200-300 g) obtained from the experimental animal center of Central South University (Changsha, China) were maintained in a constant temperature and humidity environment under specific pathogen-free conditions, with 12-hour light/dark cycles and free access to food and water.

Cardiac fibrosis model rats were established by subcutaneously injected with ISO $(10 \mathrm{mg} / \mathrm{kg})$ per day for 2 weeks. ISO-induced cardiac fibrosis rats were divided into two groups, eight in each group. Rats were injected with $100 \mu \mathrm{g}(100 \mu \mathrm{L})$ HAFMSCExos via the tail vein. In exosomes treatment group rats. Meanwhile, the same volume of PBS was given to rats in the control group (PBS). The exosomes or PBS injection was performed after the last ISO injection, and the injection lasts for 7 days.

The tissue of the heart was harvested 7 days after the exosomes or PBS treatment. All rats were deeply anesthetized with chloral hydrate intraperitoneal injection and perfused transcardially with PBS containing heparin. Then, $4 \%$ of paraformaldehyde was used to fix the brain tissues at $4{ }^{\circ} \mathrm{C}, \mathrm{pH}$ 7.4. Hearts were removed from the body and postfixed in $4 \%$ paraformaldehyde for $24 \mathrm{~h}$. The extracted heart tissue was dehydrated in a gradient sucrose solution $(10 \%, 20 \%$, and $30 \%)$.

\section{Exosomes uptake assay in vivo}

Exosomes uptake assay was performed to evaluate whether HAFMSCExos can be absorbed in the heart of rats in vivo. The PKH26 dye (PKH26, Sigma) The PKH26 dye was selected to label the lipid membranes with a red fluorescent as previously described (29). For exosomes uptake assay by rats' hearts in vivo, $400 \mu \mathrm{g}$ PKH26-labeled HAFMSCExos was injected via the tail vein in healthy adult SD rats. $12 \mathrm{~h}$ after the injection of the exosome, all rats were deeply anesthetized with pentobarbitone intraperitoneal injection, and perfused transcardially with PBS containing heparin., and the heart was harvested. The left ventricle was counterstained with CD31 (Invitrogen, Carlsbad, USA) and DAPI (R\&D). The PKH26-labeled exosomes uptake by heart tissue was analyzed by fluorescent signal analysis with a fluorescence microscope (Leica, Solms, Germany).

\section{Hematoxylin-eosin (HぬE) and Masson staining}

The left ventricles of the rat hearts were fixed in $4 \%$ paraformaldehyde and embedded in paraffin for $\mathrm{H} \& \mathrm{E}$ and Masson staining. The hearts were fixed for $24 \mathrm{~h}$ in $4 \%$ paraformaldehyde, then the tissue blocks of heart were dehydrated, embedded in paraffin. Finally, the left ventricles of hearts were cut into $4-\mu m$-thick slices for staining. The prepared slices of heart were heated overnight at $37^{\circ} \mathrm{C}$, then dewaxed, and stained with $\mathrm{H} \& \mathrm{E}$ and Masson trichrome according to the standard procedures.

\section{Immunofluorescence staining}

The tissue from the left ventricle was sectioned transversely into $20 \mu \mathrm{m}$ thickness serial sections after fixation and dehydration. Tissue from the left ventricle sections was mounted on glass slides for staining, and half of the sections were blocked with $5 \%$ BSA in $1 \%$ PBS at room temperature for an hour. The sections were incubated overnight at $4{ }^{\circ} \mathrm{C}$ with primary antibodies (CD31, PCNA, 1:100 R\&D). Then, the corresponding secondary antibodies and DAPI (Abcam, 1:500) were incubated for $1 \mathrm{~h}$ at room temperature. The nuclei in the left ventricle were counterstained with DAPI, and images were observed by using a fluorescence microscope (Olympus). 


\section{Western blot analysis}

The expression level of target proteins in the left ventricle of rats 14 days after treatment with exosomes or PBS was quantified by Western blotting. The target protein included hypoxia-inducible factor $1 \alpha(\mathrm{HIF}-1 \alpha)$ and vascular endothelial growth factor (VEGF). Rats were sacrificed by intraperitoneal injection of pentobarbitone. Western blotting was performed as previously reported (30). Radio immunoprecipitation assay (RIPA) lysis buffer supplemented with a protease inhibitor cocktail was selected to homogenize the left ventricle tissue. After centrifugation at $12,000 \mathrm{rpm}$ for $10 \mathrm{~min}$ at $4{ }^{\circ} \mathrm{C}$, the soluble protein was obtained. Collected the supernatant, and bicinchoninic acid (BCA) protein assay (Beyotime, China) was selected to detect the protein concentration. For immunoblotting, proteins from ventricle lysates containing equal $50 \mu \mathrm{g}$ of protein were loaded in $10 \%$ sodium dodecyl sulfatepolyacrylamide gel electrophoresis (SDS-PAGE) and then blotted onto polyvinylidene fluoride (PVDF) membranes (Merck Millipore, USA). Membranes were blocked in $5 \%$ non-fat milk dissolved in PBS at room temperature for an hour. Subsequently, membranes were incubated with primary antibodies HIF- $1 \alpha(1: 1,000$, Abcam $)$ and VEGF $\left(1: 1,000\right.$, Abcam) overnight at $4{ }^{\circ} \mathrm{C}$. glyceraldehyde 3 -phosphate dehydrogenase (GAPDH) was used as an internal control to demonstrate equal protein loading.

Subsequently, the membrane was washed three times with TBS-T. Membranes were incubated with horseradish peroxidase (HRP)-conjugated secondary IgG antibodies (1:20,000, Jackson ImmunoResearch, USA) for $1 \mathrm{~h}$ at room temperature. Followed by five TBS-T washes and one final TBS wash, and thereafter they were visualized by enhanced chemiluminescence plus system (Merck Millipore, USA). All samples were performed in duplicate. The software of Image Lab 3.0 system (Bio-Rad, USA) was used for quantification the densities of bands. Densitometry analysis was carried out using ImageJ.

\section{Microvascular imaging using micro-CT}

The microvascular imaging of the heart was assessed using micro-CT for the rats 28 days after the exosomes or PBS treatment. The micro-CT scanning was performed as described previously (31). The rats were anesthetized with $10 \%$ chloral hydrate $(0.3 \mathrm{~mL} / \mathrm{kg})$ injected intraperitoneally. The heart was exposed after thoracotomy rapidly and perfused heparinized saline to drain out the blood via the ascending aorta. $10 \%$ buffered formalin and the component contrast agents (Microfil MV-122, Flow-Tech, CA, USA) were perfused for vessel network fixation. The hearts of the rat were harvested and fixed in $10 \%$ formalin solution for $24 \mathrm{~h}$ at $4{ }^{\circ} \mathrm{C}$. Then the samples were scanned by a micro-CT apparatus (Explore Locus SP, GE Healthcare, USA).

\section{Statistical analysis}

Statistical analyses were performed using the SPSS 22.0 software (IBM Corp., USA), with data presented as the mean \pm standard deviation $(\mathrm{SD})$ of three replicates. The Shapiro-Wilk test was selected to examine the distribution of the data. Statistical comparisons between two groups were analyzed by unpaired Student's $t$-test. $\mathrm{P}<0.05$ was considered to indicate a statistically significant difference.

\section{Ethical Statement}

The study was conducted in accordance with the Declaration of Helsinki (as revised in 2013). The study was approved by the Committee on the Ethics of Xiangya Hospital, Central South University (No. 202004085) and informed consent was taken from all the patients.

Experiments were performed under a project license (No. 2020sydw0096) granted by the Committee on the Ethics of Animal Experiments of Central South University, in compliance with Central South University institutional guidelines for the care and use of animals.

\section{Results}

\section{Characterization of hAFMSCExos}

Human amniotic fluid was obtained from healthy mothers between 32- and 36-week gestation without HIV, hepatitis $\mathrm{B}$ and hepatitis $\mathrm{C}$ following informed consent. The Primary hAFMSCs were isolated and purified from the human amniotic fluid successfully. The hAFMSCs had a defined spindle-shaped fibroblastic morphology under a light microscope (Figure 1A). The multipotent nature of hAFMSCs was confirmed by adipocyte, osteoblast, or chondroblast differentiation after corresponding differentiation media culture (Figure $1 B, C, D$ ). Flow cytometric analysis was performed to characterize the phenotype of hAFMSCs, and isolated hAFMSCs positively expressed CD29, CD44, and CD105 and negatively expressed CD34 and CD45 (Figure 1E). 
A

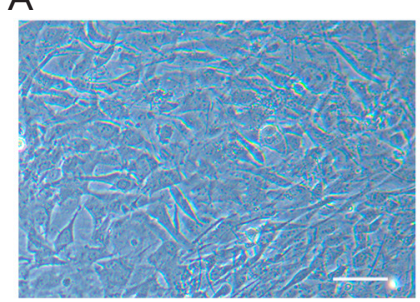

B

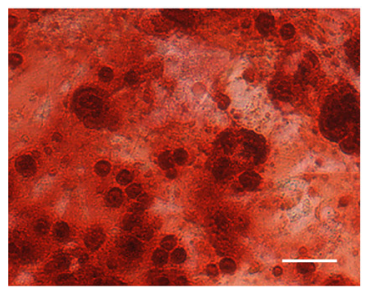

C

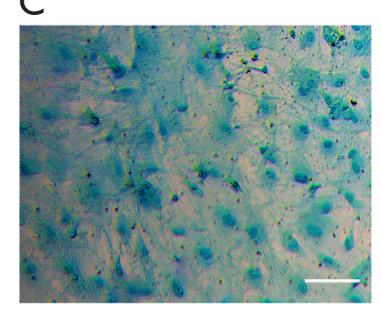

D

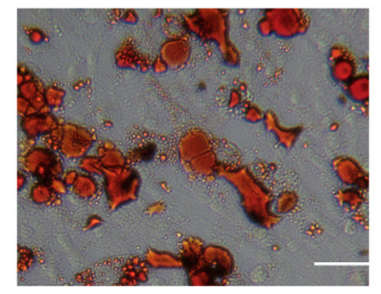

E

CD34

CD45

CD29
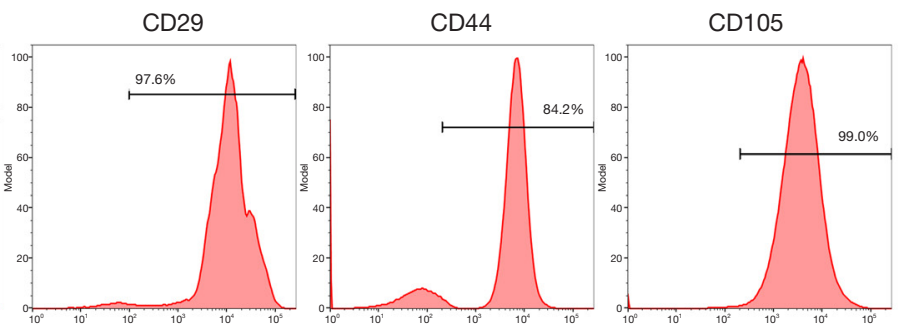

$\mathrm{F}$

G

G
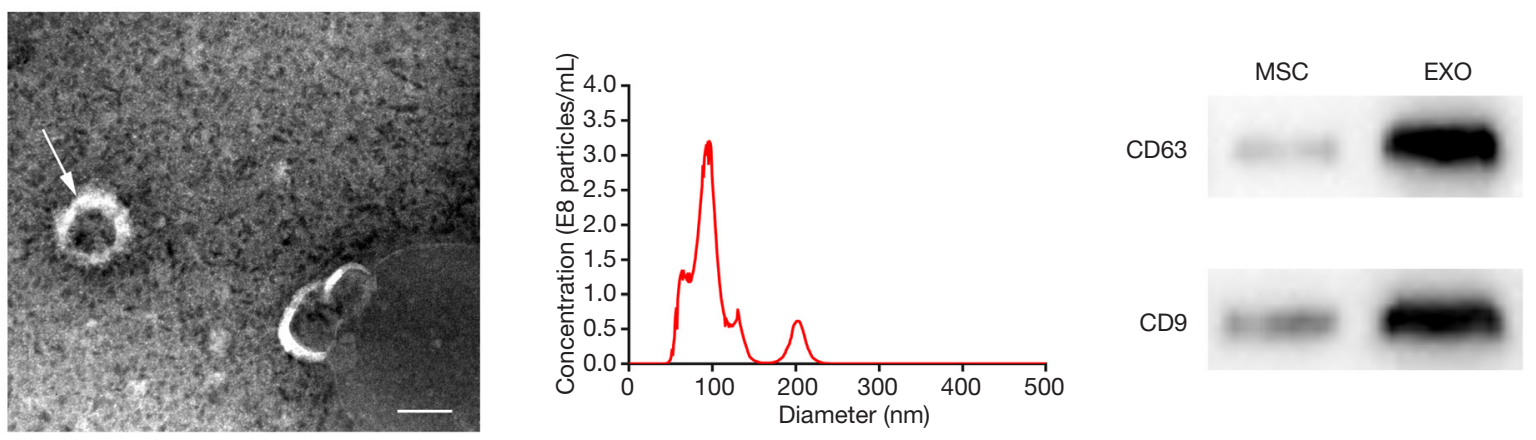

Figure 1 Characterization of hAFMSCExos. (A) The morphology of hAFMSCs under a light microscope; scale bar: 300 mm. (B,C,D) The multipotent nature of hAFMSCs was confirmed by adipocyte, osteoblast, or chondroblast differentiation after corresponding differentiation media culture, and Alizarin Red staining (B), Alcian Blue staining (C), and Oil Red O staining (D) were tested respectively; scale bar: $100 \mu \mathrm{m}$. (E) Characterize the phenotype of hAFMSCs by flow cytometric analysis. (F) The morphology of hAFMSCExos under electron microscopy; the arrow indicated the hAFMSCExos; scale bar: $100 \mathrm{~nm}$. (G) The particle size distribution of the hAFMSCExos by nanoparticle tracking analysis. (H) Traditional exosomal markers, CD9 and CD63 were tested by Western blot in the hAFMSCExos. hAFMSCExos, human amniotic fluid mesenchymal stem cell-derived exosomes; hAFMSCs, human amniotic fluid mesenchymal stem cells.

The exosomes were isolated and purified from hAFMSCs using a standard protocol described previously. Electron microscopy analysis revealed exosomes were typical spheroids or cup-shaped particles with diameters of 30 to $100 \mathrm{~nm}$ in isolated fractions (Figure $1 \mathrm{~F}$ ). Nanoparticle tracking analysis confirmed that the particle size of the hAFMSCExos mainly ranged from 30 to $100 \mathrm{~nm}$ (Figure 1G). Furthermore, Western blot showed that CD9 and CD63 were expressed in the hAFMSCExos (Figure 1H).

\section{HAFMSCExos promote migration, tube formation of HUVECs after OGD in vitro}

Fluorescence microscopy showed that PKH26-labeled exosomes were taken up into HUVECs (Figure $2 A$ ). The transwell assays (Figure $2 B, C$ ) and scratch wound healing assay (Figure 2D,E) indicated that hAFMSCExos remarkably enhance the motility and migration of HUVECs compared with the PBS. Meanwhile, tube formation assay (Figure $2 F, G$ ) indicated that the hAFMSCExos have more 

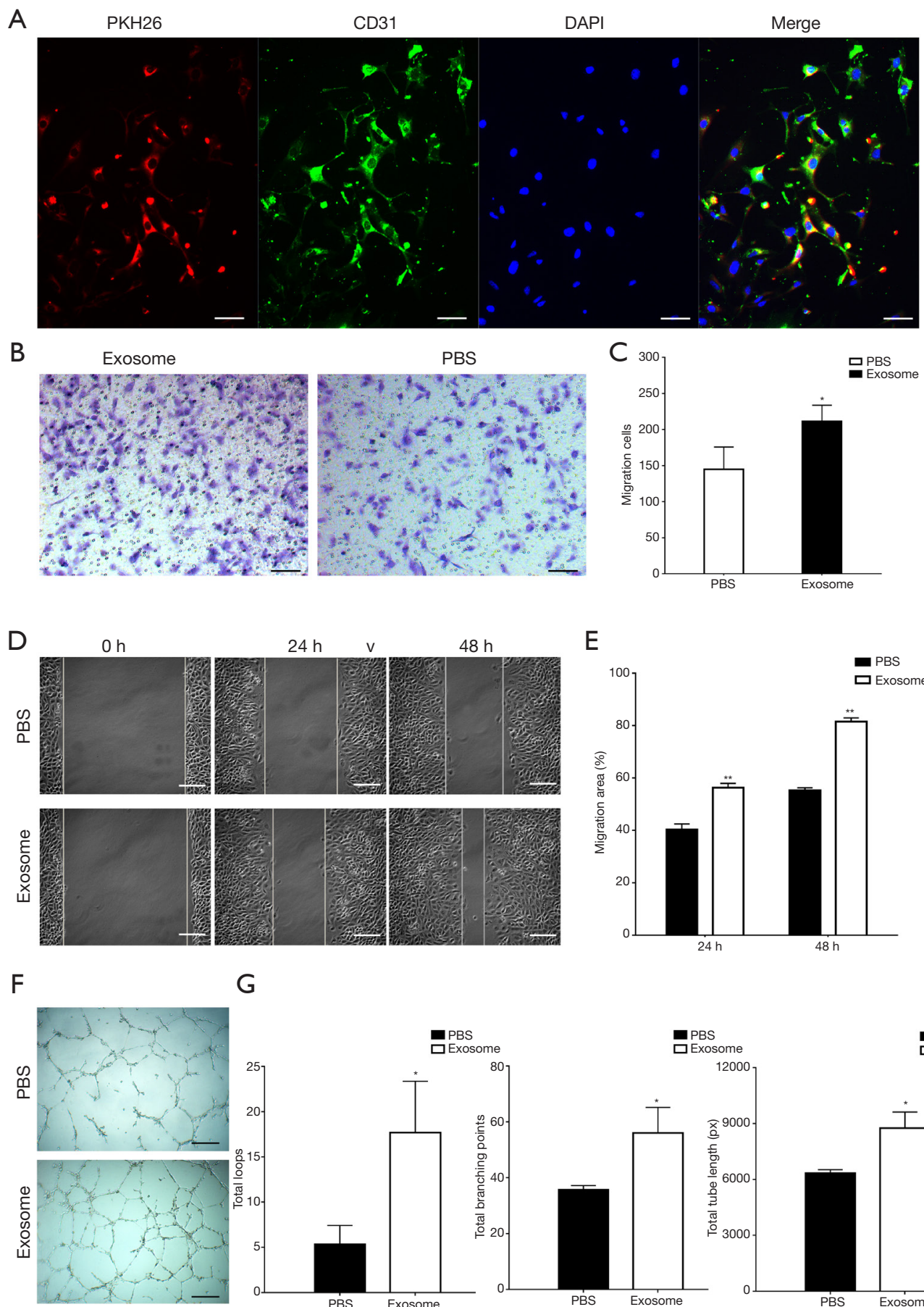

G

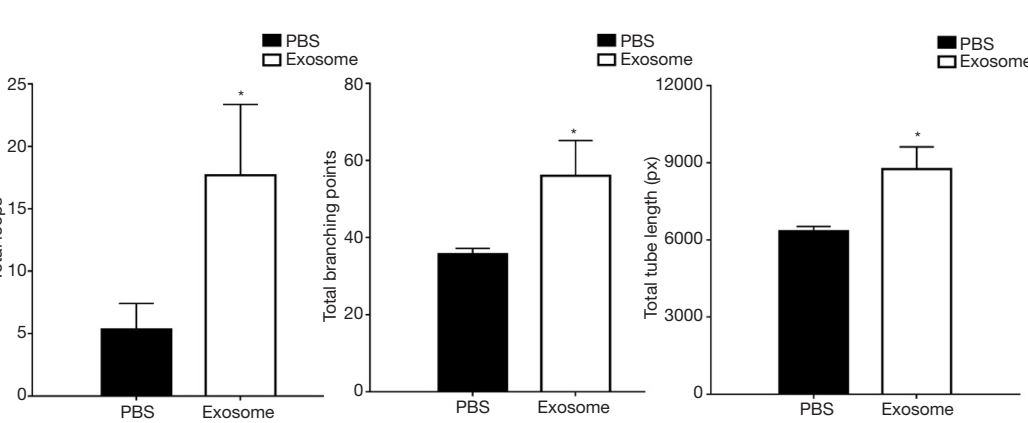

Figure 2 The hAFMSCExos promoted migration and tube formation of HUVECs after OGD in vitro. (A) HUVECs uptaken PKH26-labeled hAFMSCExo by fluorescence microscopy analysis; scale bar: $100 \mu \mathrm{m}$. (B,C) The migration of HUVECs stimulated by hAFMSCExos or PBS after OGD was analyzed by transwell assay; scale bar: $100 \mu \mathrm{m}$; *, $\mathrm{P}<0.05$. (D,E) The migration of HUVECs treated with hAFMSCExos or PBS after OGD in scratch wound assay; scale bar: $50 \mu \mathrm{m} ;{ }^{* *}, \mathrm{P}<0.01$. (F) The tube formation ability of treated with hAFMSCExos or PBS after OGD in capillary network formation assay; scale bar: $50 \mu \mathrm{m}$. (G) The total loops, total branching points, and total tube length of HUVECs treated with hAFMSCs-Exos or PBS after OGD in capillary network formation assay. *, P<0.05; hAFMSCExos, human amniotic fluid mesenchymal stem cell-derived exosomes; HUVEC, human umbilical vein endothelial cells; OGD, oxygen and glucose deprivation; PBS, phosphate-buffered saline. 
capillary-like structures compared with PBS group. Besides, quantification of total tube length, total branching points and total loops were significantly raised in HUVECs treated with hAFMSCExos. As evidenced by these above assays, our research suggests that hAFMSCExos could enhance proangiogenic effects on HUVEC endothelial cells.

\section{The bAFMSCExos promote angiogenesis after ISO induced cardiac fibrosis in rats from two-dimensional (2D) to three-dimensional (3D)}

All rats were accepted the treatment as the experiment protocol. HAFMSCExos were labeled with PKH26 dye, and immunofluorescence showed that exosomes were uptaken (Figure 3A). H\&E and Masson staining showed that the difference of cardiac fibrosis degree in Exosome and PBS groups, and staining suggested the exosomes relieve the ECM deposition in ISO-induced cardiac fibrosis than PBS (Figure 3B). The protein levels of Collagen I and $\alpha$-SMA were lower in exosomes group rats than the PBS group $(\mathrm{P}<0.001$ and $\mathrm{P}<0.005$, respectively, Figure $3 C, D)$. Immunofluorescence was also selected to evaluate the vascular regeneration information after treatment with exosomes or PBS. CD31 staining analysis was used to detect angiogenesis at 7 days after exosomes or PBS treatment, and results suggested that hAFMSCExos can promote the expression of CD31 in the rats (Figure $3 E$ ). Meanwhile, the number of regenerated microvessels was significantly enhanced in rats administrated with exosomes by quantitative analysis of microvessel density (Figure 3F). Furthermore, the micro-CT scanning was performed to test the $3 \mathrm{D}$ microvascular networks at 7 days after exosomes or PBS treatment post-MI (Figure 3G). The 3D microvascular reconstruction networks evidenced that hAFMSCExos promote angiogenesis after cardiac fibrosis.

\section{The hAFMSCExos enhanced the expressions of HIF-1a and VEGF after ISO induced cardiac fibrosis rats}

The protein levels of HIF-1 $\alpha$ and VEGF expression in the left ventricle accepted hAFMSCExos were higher than PBS treatment at 7 days post-treatment by Western blot analysis (Figure 4A,B).

\section{Discussion}

In the present study, we demonstrated that hAFMSCExos promoted tube formation, migration, angiogenesis of HUVECs after OGD, alleviated ECM deposition, promoted angiogenesis and further accompanied by enhanced the expression levels of HIF-1 $\alpha$ and VEGF in ISO induced cardiac fibrosis rats.

Cardiac fibrosis is a pathological process mainly characterized as excess ECM deposition (1), is contributing to the presence and development in nearly all types of heart disease (2), has been a powerful predictor of death and adverse cardiac events in HF patients $(3,4)$. ECM deposition and CFs transdifferentiation play a critical role in cardiac fibrosis. Both the quality and quantity of the ECM composition were dramatically changed during cardiac fibrosis, which may impair the heart physically and electrically, vastly reducing cardiac function. Meanwhile, myofibroblast, as a secretory and contractile cell, can not only synthesize collagen fibers, but also have contraction function, express excessive ECM protein, protease, vimentin, desmin, myosin, and $\alpha$-smooth muscle actin ( $\alpha$-SMA) (32). Moreover, myofibroblasts are factories of ECM protein production (33). Myofibroblasts produce excessive ECM proteins leading to the expansion of cardiac interstitium during the repair of damaged cardiomyocytes (34). Cardiac fibrosis was significantly attributed to activation and excessive proliferation of CFs $(5,6)$.

The adult mammalian heart has a poor regenerative ability; thus, the damage and loss of numerous cardiomyocytes trigger the fibroblasts to activate into myofibroblasts and start the reparative program. Unfortunately, no effective medication treatment could rescue the fibrotic cardiac tissue, due to the lack of selfregeneration capability of the cardiomyocyte. It is urgent to pursue effective therapeutic strategies for cardiac fibrosis. Recent literature has shown that extracellular vesicles (EVs), especially exosomes may also modify cardiac repair and the fate of fibrosis in cardiac fibrosis $(19,20)$.

MSCs are a promising therapeutic candidate in cellbased therapy in cardiac fibrosis treatment contribute to the advantages of inflammatory response alleviation, myocardial perfusion improvement, cardiomyocyte apoptosis prevention, and angiogenesis promotion (35-37). Research indicated that exosomes were the initiating agent of the therapeutic effect of MSCs (7-10). Exosomes as nanometer-sized (40-100 $\mathrm{nm}$ diameter) vesicles, play a critical role in intercellular communication. Exosomes have been frequently investigated in pathophysiological processes including cardiovascular diseases $(38,39)$. Exosomes were regarded as an important regulatory factor in cardiovascular 
A

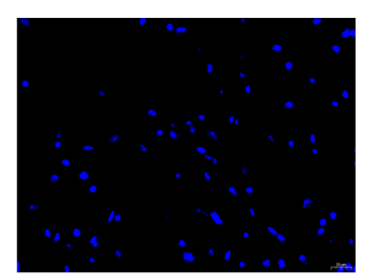

B

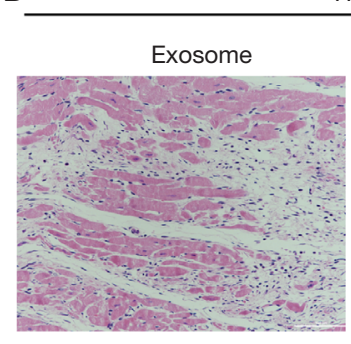

C

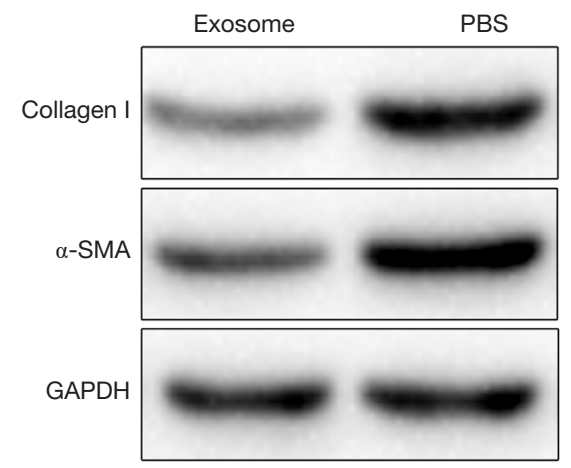

E

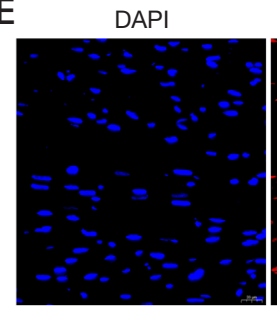

DAPI

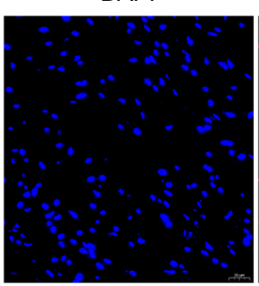

CD31

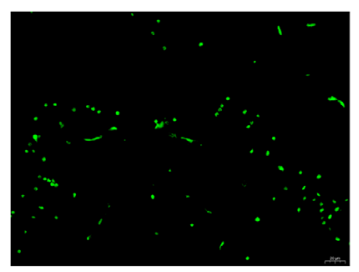

H\&E

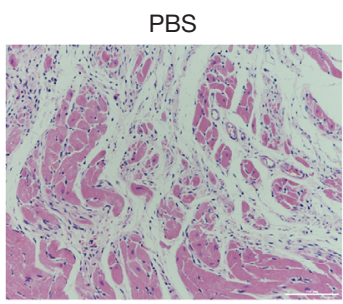

BS

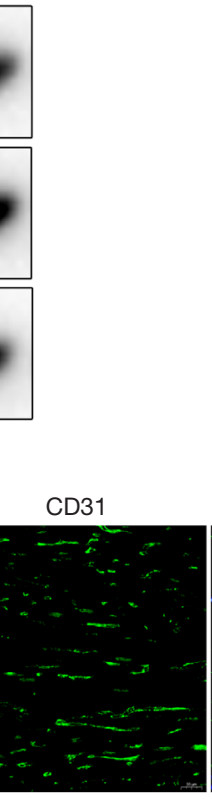

CD31
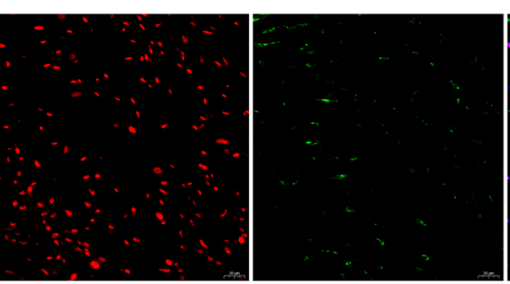

PHK26

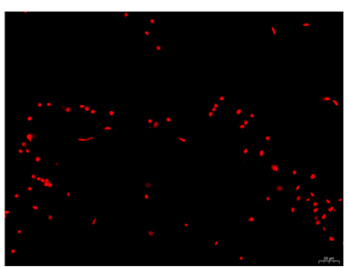

MASSON

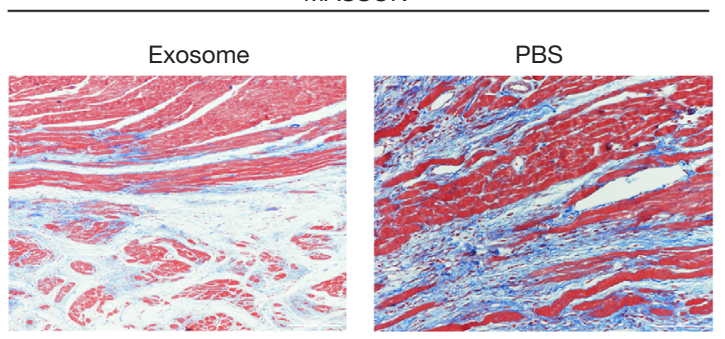

D

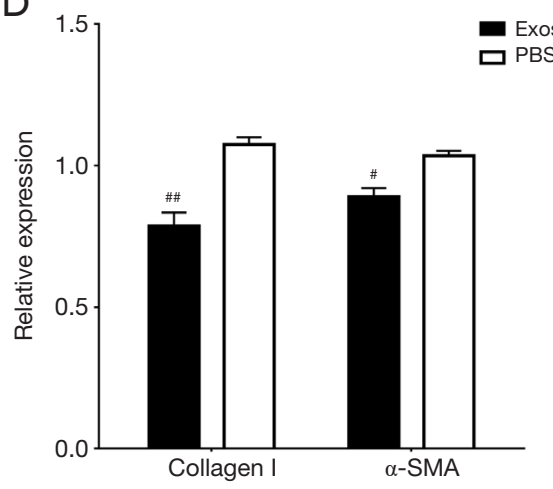

Exosome

PBS

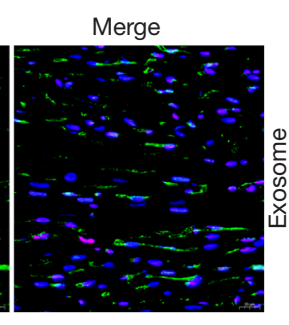

Merge
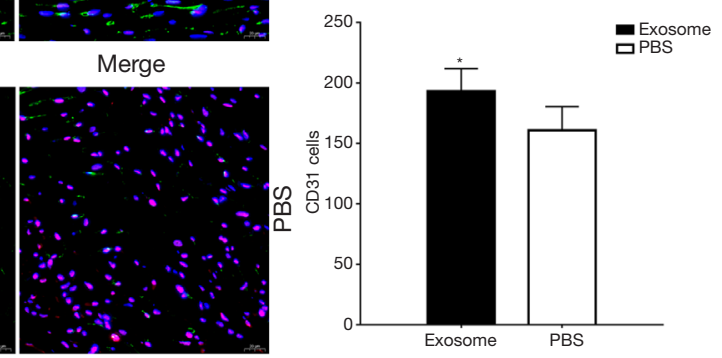

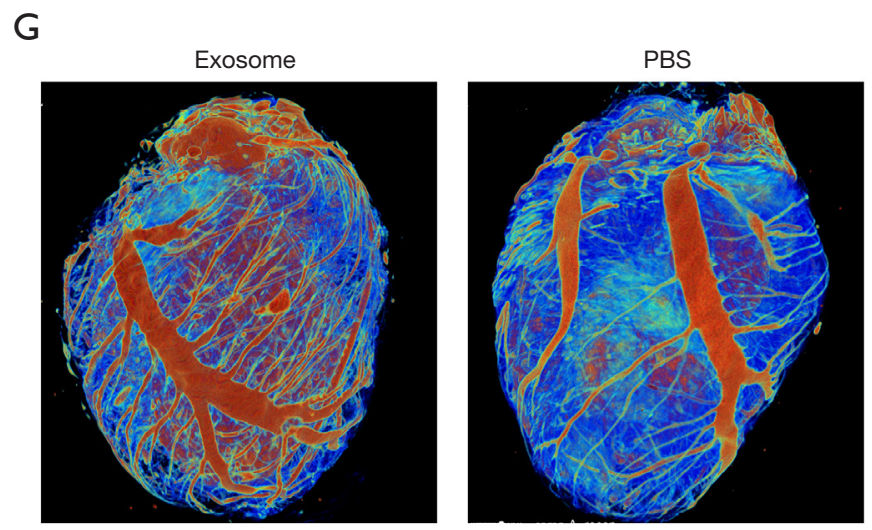

Figure 3 The hAFMSCs-Exos promote angiogenesis after ISO-induced cardiac fibrosis in rats. (A) PKH26-labeled hAFMSCs-Exos were uptaken by vascular endothelium cell after ISO-induced cardiac fibrosis in rats; scale bar: $20 \mu \mathrm{m}$. (B) The levels of cardiac fibrosis in rats treated with hAFMSCExos or PBS by H\&E and Masson staining; scale bar:100 $\mu \mathrm{m}$. (C) The protein levels of Collagen I and $\alpha$-SMA in rats treated with hAFMSCExos or PBS by Western blot. (D) Quantitative analysis of the protein levels of Collagen I and $\alpha$-SMA in rats

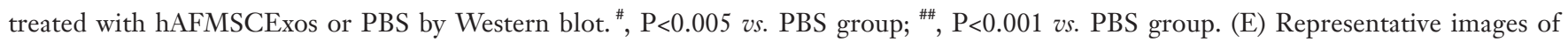
PCNA-positive (red) and CD31 positive (green) cells in the left ventricle in rats treated with hAFMSCExos or PBS; scale bar: $20 \mu \mathrm{m}$. (F) Quantitative analysis of CD31 positive cells in the left ventricle in rats treated with hAFMSCExos or PBS. *, $\mathrm{P}<0.05$ vs. PBS (control) group (G) Microvascular regeneration 14 days post-treatment with hAFMSCExos or PBS. hAFMSCExos, human amniotic fluid mesenchymal stem cell-derived exosomes; PBS, phosphate-buffered saline; H\&E, hematoxylin-eosin.

disease including cardiomyocyte hypertrophy, MI, apoptosis, cardiac fibrosis, ischemia, and angiogenesis $(40,41)$. The effect of exosomes in anti-apoptotic, antifibrotic, and pro-angiogenic roles enhanced cardiac repair and cardiomyocyte regeneration (12-15).

Exosome shows great promise in cardiovascular disease treatment because of its high biocompatibility and low immunogenicity, its ability mainly consisted of enhance angiogenesis, alleviate cardiomyocyte apoptosis, and antcardiac fibrosis (42). Exosomes may act as a potential biomarker and therapeutic agent in cardiac fibrosis. Exosomes exert great potential in anti-cardiac fibrosis in several pieces of research. The research suggested that TIMP2-modified human umbilical cord MSC-derived exosomes ameliorate MI injury, alleviate myocardial apoptosis and anti-cardiac fibrosis (43). Research indicated that exosomes show great promise in reduce myocardial fibrosis (42). Meanwhile, exosomes have anti-apoptosis, anti-fibrosis, and angiogenesis effects on infracted cardiomyocytes and may be novel targets for treating MI (13-15). Our research demonstrated that hAFMSCExos alleviated the degree of ISO-induced cardiac fibrosis. Moreover, exosomes can offer effective therapeutic effects without engraftment requirement, which reduces concerns for tumorigenesis (16).
Current research successfully obtained and isolated hAFMSCExos from leftover samples of amniotic fluid cesarean section (25). Recent research showed that hAFMSCs can provide higher exosomes productivity than bone marrow MSCs with similar morphology and biological characteristics (16). Moreover, HAFMSCs exhibit inordinately high self-renewal and can be industrialized more easily than other MSCs $(29,44)$. The human amniotic fluid is easy to obtain without ethical concerns.

A recent study has demonstrated that hAFMSCExos had more paracrine factors, including VEGF and TGF- $\beta 1$, and secretes enhanced the proliferation and migration of human dermal fibroblasts both in vitro and in vivo (45). Our current research indicated that hAFMSCExos not only alleviated cardiac fibrosis but also enhanced the number of CD31immunoreactive cells and the level of HIF-1 $\alpha$ expression in ISO-induced rats' hearts. We also found that hAFMSCExos promoted tube formation, migration, angiogenesis of HUVECs in vivo.

Angiogenesis, which plays a critical role in the ischemic microenvironment recovery, highly count on the migration and proliferation of vascular endothelial cells. The research reported that human umbilical cord MSC-derived exosomes (46), bone marrow MSC-derived exosomes (47), and cardiac MSC-derived exosomes (48) accelerated 
A

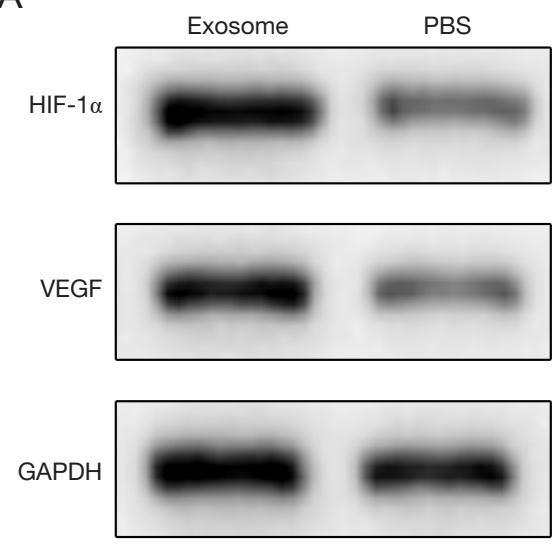

B

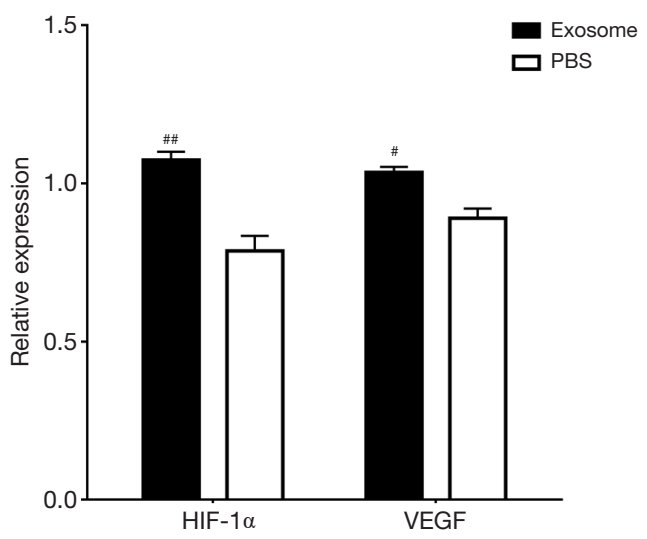

Figure 4 The expressions of HIF-1 $\alpha$ and VEGF in the left ventricle after accepted hAFMSCExos or PBS treatment. (A) The levels of HIF$1 \alpha$ and VEGF expression in the left ventricle after accepted hAFMSCExos or PBS treatment by Western blot analysis. (B) Quantitative analysis showed that the expressions of HIF- $1 \alpha$ and VEGF in the left ventricle after accepted hAFMSCExos or PBS treatment by Western blot analysis. ", $\mathrm{P}<0.005$ vs. PBS group; ;, $\mathrm{P}<0.001$ vs. PBS group. HIF-1 $\alpha$, hypoxia-inducible factor $1 \alpha$; VEGF, vascular endothelial growth factor; hAFMSCExos, human amniotic fluid mesenchymal stem cell-derived exosomes; PBS, phosphate-buffered saline.

angiogenesis. Another research demonstrated that TIMP2modified human umbilical cord MSC-derived exosomes increased CD31-immunoreactive cells expression which indicated the angiogenesis in the myocardium of MI rats, meanwhile it promoted the migration, proliferation, and tube forming ability of HUVECs in vitro (43).

Research has confirmed that angiogenesis by HIF- $1 \alpha$ plays a critical role in anti-cardiac fibrosis. HIF- $1 \alpha$ has been taken as the most important transcriptional regulator in response to hypoxia (49). Current studies reported that HIF- $1 \alpha$ directly regulates the expression of genes involved in angiogenesis (42,50-52), which including VEGF, platelet-derived growth factor (PDGF), and angiopoietin 1 (Ang-1) (53). The research demonstrated HIF-1 $\alpha-$ overexpressed exosomes had a cardioprotective effect on MI rat via enhanced angiogenesis and reduced fibrosis, there were promotional effects on proliferation, migration, and tube forming ability on HUVECs (54). Evidence has elucidated that HIF- $1 \alpha$ regulated the expression of VEGF at the transcription level (55). Moreover, evidence indicated that increased capillary density induced by HIF- $\alpha$ was as well as VEGF expression in peri-infarct and infarct regions of MI heart $(55,56)$.

\section{Conclusions}

Together, our studies demonstrated that hAFMSCExos have proangiogenic effects on endothelial cells and enhanced angiogenesis in the cardiac fibrosis model. The hAFMSCExos may contribute to the enhanced expressions of HIF-1 and VEGF in the left ventricle of the cardiac fibrosis rats. The hAFMSCExos may be a potential treatment strategy for cardiac fibrosis.

\section{Acknowledgments}

We thank the Department of medical ultrastructure, School of Basic Medicine, and the Lab of Biomedical Electronic Microscopy of Higher Research Center, Central South University for assistance with TEM work. We would be grateful to Junpu Wang for his help of making EM sample. Funding: This work was supported by the Natural Science Foundation of Hunan Province (2019JJ50936 to PL, 2019JJ50950 to CZ, 2019JJ50954 to XC), and the Youth Science Foundation of Xiangya Hospital (2019Q14 to CZ).

\section{Footnote}

Reporting Checklist: The authors have completed the ARRIVE reporting checklist. Available at http://dx.doi. org/10.21037/cdt-20-1032

Data Sharing Statement: Available at http://dx.doi. org/10.21037/cdt-20-1032

Conflicts of Interest: All authors have completed the ICMJE 
uniform disclosure form (available at http://dx.doi. org/10.21037/cdt-20-1032). The authors have no conflicts of interest to declare.

Ethical Statement: The authors are accountable for all aspects of the work in ensuring that questions related to the accuracy or integrity of any part of the work are appropriately investigated and resolved. The study was conducted in accordance with the Declaration of Helsinki (as revised in 2013). The study was approved by the Committee on the Ethics of Xiangya Hospital, Central South University (No. 202004085) and informed consent was taken from all the patients. Experiments were performed under a project license (No. 2020sydw0096) granted by the Committee on the Ethics of Animal Experiments of Central South University, in compliance with Central South University institutional guidelines for the care and use of animals.

Open Access Statement: This is an Open Access article distributed in accordance with the Creative Commons Attribution-NonCommercial-NoDerivs 4.0 International License (CC BY-NC-ND 4.0), which permits the noncommercial replication and distribution of the article with the strict proviso that no changes or edits are made and the original work is properly cited (including links to both the formal publication through the relevant DOI and the license). See: https://creativecommons.org/licenses/by-nc-nd/4.0/.

\section{References}

1. Zhang C, Zhang $\mathrm{Y}$, Zhu H, et al. MiR-34a/miR-93 target c-Ski to modulate the proliferaton of rat cardiac fibroblasts and extracellular matrix deposition in vivo and in vitro. Cell Signal 2018;46:145-53.

2. Porter KE, Turner NA. Cardiac fibroblasts: at the heart of myocardial remodeling. Pharmacol Ther 2009;123:255-78.

3. Ma CX, Zhao XK, Li YD. New therapeutic insights into radiation-induced myocardial fibrosis. Ther Adv Chronic Dis 2019;10:2040622319868383.

4. Gulati A, Japp AG, Raza S, et al. Absence of myocardial fibrosis predicts favorable long-term survival in new-onset heart failure. Circ Cardiovasc Imaging 2018;11:e007722.

5. Ali SR, Ranjbarvaziri S, Talkhabi M, et al. Developmental heterogeneity of cardiac fibroblasts does not predict pathological proliferation and activation. Circ Res 2014;115:625-35.

6. Travers JG, Kamal FA, Robbins J, et al. Cardiac fibrosis: the fibroblast awakens. Circ Res 2016;118:1021-40.

7. Fang Y, Zhang Y, Zhou J, et al. Adipose-derived mesenchymal stem cell exosomes: a novel pathway for tissues repair. Cell Tissue Bank 2019;20:153-61.

8. Li JJ, Hosseini-Beheshti E, Grau GE, et al. Stem cellderived extracellular vesicles for treating joint injury and osteoarthritis. Nanomaterials (Basel) 2019;9:261 .

9. Mendt M, Rezvani K, Shpall E. Mesenchymal stem cellderived exosomes for clinical use. Bone Marrow Transplant 2019;54:789-92.

10. Roşca AM, Țuţuianu R, Titorencu ID. Mesenchymal stromal cells derived exosomes as tools for chronic wound healing therapy. Rom J Morphol Embryol 2018;59:655-62.

11. Park KS, Bandeira E, Shelke GV, et al. Enhancement of therapeutic potential of mesenchymal stem cell-derived extracellular vesicles. Stem Cell Res Ther 2019;10:288.

12. Pan J, Alimujiang $M$, Chen Q, et al. Exosomes derived from miR-146a-modified adipose-derived stem cells attenuate acute myocardial infarction-induced myocardial damage via downregulation of early growth response factor 1. J Cell Biochem 2019;120:4433-43.

13. Singla DK. Stem cells and exosomes in cardiac repair. Curr Opin Pharmacol 2016;27:19-23.

14. Mincheva-Nilsson L, Baranov V, Nagaeva O, et al. Isolation and characterization of exosomes from cultures of tissue explants and cell lines. Curr Protoc Immunol. 2016;115:14.42.1-14.42.21.

15. Khan M, Nickoloff E, Abramova T, et al. Embryonic stem cell-derived exosomes promote endogenous repair mechanisms and enhance cardiac function following myocardial infarction. Circ Res 2015;117:52-64.

16. Tracy SA, Ahmed A, Tigges JC, et al. A comparison of clinically relevant sources of mesenchymal stem cellderived exosomes: bone marrow and amniotic fluid. J Pediatr Surg 2019;54:86-90.

17. Chen CC, Liu L, Ma F, et al. Elucidation of exosome migration across the blood-brain barrier model in vitro. Cell Mol Bioeng 2016;9:509-29.

18. Phinney DG, Pittenger MF. Concise review: MSC-derived exosomes for cell-free therapy. Stem Cells 2017;35:851-8.

19. Barile L, Milano G, Vassalli G. Beneficial effects of exosomes secreted by cardiac-derived progenitor cells and other cell types in myocardial ischemia. Stem Cell Investig 2017;4:93.

20. Wang C, Zhang C, Liu L, et al. Macrophage-derived mir155-containing exosomes suppress fibroblast proliferation and promote fibroblast inflammation during cardiac injury. Mol Ther 2017;25:192-204. 
21. Aryani A, Denecke B. Exosomes as a nanodelivery system: a key to the future of neuromedicine? Mol Neurobiol 2016;53:818-34.

22. Maia J, Caja S, Strano MM, et al. Exosome-based cell-cell communication in the tumor microenvironment. Front Cell Dev Biol 2018;6:18.

23. Wu X, Wang Y, Xiao Y, et al. Extracellular vesicles: potential role in osteoarthritis regenerative medicine. J Orthop Translat 2019;21:73-80.

24. De Coppi P, Bartsch GJ, Siddiqui MM, et al. Isolation of amniotic stem cell lines with potential for therapy. Nat Biotechnol 2007;25:100-6.

25. Schiavo AA, Franzin C, Albiero M, et al. Endothelial properties of third-trimester amniotic fluid stem cells cultured in hypoxia. Stem Cell Res Ther 2015;6:209.

26. Bollini S, Cheung KK, Riegler J, et al. Amniotic fluid stem cells are cardioprotective following acute myocardial infarction. Stem Cells Dev 2011;20:1985-94.

27. Zhang Z, Yang J, Yan W, et al. Pretreatment of cardiac stem cells with exosomes derived from mesenchymal stem cells enhances myocardial repair. J Am Heart Assoc 2016;5:e002856.

28. Wang N, Chen C, Yang D, et al. Mesenchymal stem cells-derived extracellular vesicles, via miR-210, improve infarcted cardiac function by promotion of angiogenesis. Biochim Biophys Acta Mol Basis Dis 2017;1863:2085-92.

29. Klein JD, Fauza DO. Amniotic and placental mesenchymal stem cell isolation and culture. Methods Mol Biol 2011;698:75-88.

30. Rocha-Ferreira E, Sisa C, Bright S, et al. Curcumin: novel treatment in neonatal hypoxic-ischemic brain injury. Front Physiol 2019;10:1351.

31. Hu J, Cao Y, Wu T, et al. Micro-CT as a tool to investigate the efficacy of tetramethylpyrazine in a rat spinal cord injury model. Spine (Phila Pa 1976) 2016;41:1272-8.

32. Kawaguchi M, Takahashi M, Hata T, et al. Inflammasome activation of cardiac fibroblasts is essential for myocardial ischemia/reperfusion injury. Circulation 2011;123:594-604.

33. van der Slot AJ, Zuurmond AM, van den Bogaerdt AJ, et al. Increased formation of pyridinoline cross-links due to higher telopeptide lysyl hydroxylase levels is a general fibrotic phenomenon. Matrix Biol 2004;23:251-7.

34. Ranjan P, Kumari R, Verma SK. Cardiac fibroblasts and cardiac fibrosis: precise role of exosomes. Front Cell Dev Biol 2019;7:318.

35. Singh A, Singh A, Sen D. Mesenchymal stem cells in cardiac regeneration: a detailed progress report of the last
6 years (2010-2015). Stem Cell Res Ther 2016;7:82.

36. Majka M, Sulkowski M, Badyra B, et al. Concise review: mesenchymal stem cells in cardiovascular regeneration: emerging research directions and clinical applications. Stem Cells Transl Med 2017;6:1859-67.

37. Kobayashi K, Suzuki K. Mesenchymal stem/stromal cellbased therapy for heart failure- what is the best source? Circ J 2018;82:2222-32.

38. Martínez MC, Andriantsitohaina R. Extracellular Vesicles in Metabolic Syndrome. Circ Res 2017;120:1674-86.

39. Jansen F, Nickenig G, Werner N. Extracellular vesicles in cardiovascular disease: potential applications in diagnosis, prognosis, and epidemiology. Circ Res 2017;120:1649-57.

40. Waldenström A, Ronquist G. Role of exosomes in myocardial remodeling. Circ Res 2014;114:315-24.

41. Sahoo S, Klychko E, Thorne T, et al. Exosomes from human CD34(+) stem cells mediate their proangiogenic paracrine activity. Circ Res 2011;109:724-8.

42. Ma T, Chen Y, Chen Y, et al. MicroRNA-132, delivered by mesenchymal stem cell-derived exosomes, promote angiogenesis in myocardial infarction. Stem Cells Int 2018;2018:3290372.

43. Ni J, Liu X, Yin Y, et al. Exosomes derived from TIMP2modified human umbilical cord mesenchymal stem cells enhance the repair effect in rat model with myocardial infarction possibly by the Akt/Sfrp2 pathway. Oxid Med Cell Longev 2019;2019:1958941.

44. Spitzhorn LS, Rahman MS, Schwindt L, et al. Isolation and molecular characterization of amniotic fluid-derived mesenchymal stem cells obtained from caesarean sections. Stem Cells Int 2017;2017:5932706.

45. Hill MF, Singal PK. Right and left myocardial antioxidant responses during heart failure subsequent to myocardial infarction. Circulation 1997;96:2414-20.

46. Zhang B, Wu X, Zhang X, et al. Human umbilical cord mesenchymal stem cell exosomes enhance angiogenesis through the Wnt4/beta-catenin pathway. Stem Cells Transl Med 2015;4:513-22.

47. Bian S, Zhang L, Duan L, et al. Extracellular vesicles derived from human bone marrow mesenchymal stem cells promote angiogenesis in a rat myocardial infarction model. J Mol Med (Berl) 2014;92:387-97.

48. Ju C, Shen Y, Ma G, et al. Transplantation of cardiac mesenchymal stem cell-derived exosomes promotes repair in ischemic myocardium. J Cardiovasc Transl Res 2018;11:420-8.

49. Heil M, Eitenmuller I, Schmitz-Rixen T, et al. Arteriogenesis versus angiogenesis: similarities and 
differences. J Cell Mol Med 2006;10:45-55.

50. Cao R, Brakenhielm E, Pawliuk R, et al. Angiogenic synergism, vascular stability and improvement of hindlimb ischemia by a combination of PDGF-BB and FGF-2. Nat Med 2003;9:604-13.

51. Carmeliet P, Jain RK. Angiogenesis in cancer and other diseases. Nature 2000;407:249-57.

52. Ceradini DJ, Kulkarni AR, Callaghan MJ, et al. Progenitor cell trafficking is regulated by hypoxic gradients through HIF-1 induction of SDF-1. Nat Med 2004;10:858-64.

53. Li M, Cui Y, He W, et al. Effects of triple-mutated hypoxia-inducible factor-1alpha on angiogenesis and cardiac function improvement in rats with myocardial

Cite this article as: $\mathrm{Hu} \mathrm{J}$, Chen X, Li P, Lu X, Yan J, Tan H, Zhang C. Exosomes derived from human amniotic fluid mesenchymal stem cells alleviate cardiac fibrosis via enhancing angiogenesis in vivo and in vitro. Cardiovasc Diagn Ther 2021;11(2):348-361. doi: 10.21037/cdt-20-1032 infarction. Cell Physiol Biochem 2018;50:2329-40.

54. Sun J, Shen H, Shao L, et al. HIF-1alpha overexpression in mesenchymal stem cell-derived exosomes mediates cardioprotection in myocardial infarction by enhanced angiogenesis. Stem Cell Res Ther 2020;11:373.

55. Koyasu S, Kobayashi M, Goto Y, et al. Regulatory mechanisms of hypoxia-inducible factor 1 activity: two decades of knowledge. Cancer Sci 2018;109:560-71.

56. Gonzalez-King H, Garcia NA, Ontoria-Oviedo I, et al. Hypoxia inducible factor-1alpha potentiates jagged 1-mediated angiogenesis by mesenchymal stem cellderived exosomes. Stem Cells 2017;35:1747-59. 

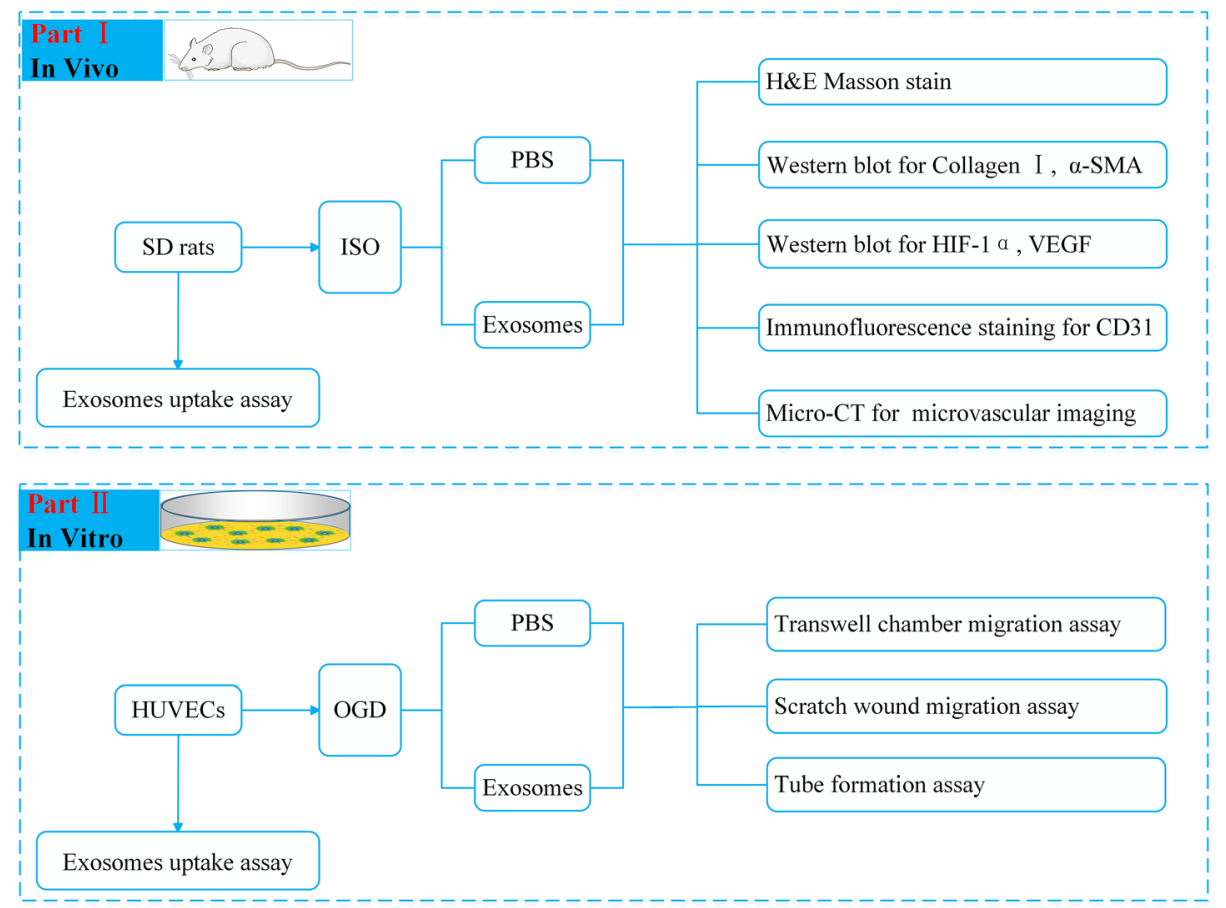

Figure S1 Process of experiments. ISO, isoproterenol; PBS, phosphate-buffered saline; H\&E, hematoxylin-eosin; $\alpha$-SMA, $\alpha$-smooth muscle actin; HIF-1 $\alpha$, hypoxia-inducible factor $1 \alpha$; VEGF, vascular endothelial growth factor; HUVEC, human umbilical vein endothelial cells; OGD, oxygen and glucose deprivation. 